\title{
tratamiento de los edificios contra la humedad del terreno
}

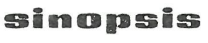

Se indican en el presente artículo los principales daños que puede producir el exceso de humedad en los materiales de construcción, estableciendo, como una de las causas fundamentales, la ascensión capilar del agua del suelo a través de cimientos y muros.

El agua existente en el terreno puede ser de dos tipos (dispersa y freática) y se dan criterios para su reconocimiento.

A continuación se indican, a título informativo, los diferentes procedimientos que se utilizan para defenderse de este tipo de humedades,

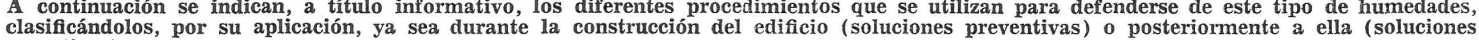
curvativas).

\section{Bomsideraciones provions}

Una de las características fundamentales a tener en cuenta en el juicio sobre una edificación, es el de su aptitud para ser ocupada de acuerdo con los fines particulares que se le asignen. Cuando se trata de construcciones destinadas a ser ocupadas por seres vivos, el conjunto de factores y circunstancias que determinan esta aptitud se denomina habitabilidad.

Entre las causas que disminuyen y pueden incluso anular dicha habitabilidad, está la excesiva humedad interior de los edificios. Para los seres humanos se considera idónea una humedad relativa que oscile entre el 40 y el 70 por 100 .

Si una edificación sobrepasa en demasía estos valores pueden derivarse una serie de daños, que es posible agrupar, según afecten a los siguientes dominios:

\section{Estética}

- eflorescencias;

- decoloración y manchas sobre las pinturas;

- formación de ampollas y desprendimiento de pinturas y revestimientos;

- mohos.

\section{Resistencia de los materiales}

- reblandecimiento de los revestimientos de yeso;

- deterioro de los revestimientos de cal;

- alabeo y variaciones dimensionales de la madera;

- podredumbre de la madera;

- desencolado de paneles y contrachapados;

- corrosión de metales;

- disgregación por el hielo de los materiales heladizos.

\section{Confort}

- olores de humedad;

- disminución de la resistencia térmica de los muros (con lo cual aumentan las pérdidas caloríficas y baja la temperatura interior):

- insalubridad general. 
De las posibles causas que pueden producir la excesiva humedad de los edificios, merece especial atención la ascensión capilar del agua del suelo a través de los cimientos y muros.

Este tipo de humedad es de los más graves, pues se manifiesta con más intensidad y permanencia que los demás.

\section{Recomocimuiento de su origen}

A diferencia de la humedad de condensación que suele atacar las últimas plantas de los modernos edificios, la humedad que proviene del subsuelo ataca con preferencia a los edificios antiguos y, salvo circunstancias excepcionales, solamente en las dos primeras plantas y sótano.

El agua existente en el terreno puede ser de dos tipos:

a) dispersa;

b) freática.

La primera es más fácil de combatir que la segunda aunque su diagnóstico presenta más dificultades, pues obedece a causas localizadas y limitadas y no a una situación uniforme y general que se traduce en una multitud de indicios.

Generalmente, se trata de aguas de lluvia defectuosamente recogidas, así como pérdidas en pozos, canalizaciones, etc., de los alrededores.

El agua suele atacar los muros a nivel del suelo o muy poco por debajo. Son muy frecuentes, por ejemplo, los casos de humedad producida por acumulación de las aguas del tejado en la base del edificio.

Para determinar el origen y causa de las humedades, se ha de proceder a una sistemática inspección del perímetro del edificio:

a) abriendo varios agujeros al pie de los muros, con objeto de descubrir si hay alguna zona seca;

b) verificando si los pozos, cisternas, cloacas circundantes presentan pérdidas;

c) inspeccionando con detenimiento si las aguas de lluvia provenientes del tejado o caídas en las inmediaciones son convenientemente alejadas;

d) poniendo al descubierto las tuberías de agua bajo presión o verificando de algún modo las eventuales pérdidas, comprobando, asimismo, si producen condensaciones.

En definitiva, el origen de la humedad se deduce de su distribución general en el edificio, que se puede determinar a partir de los análisis del \% volumétrico de humedad efectuados en la albañilería. El agua freática da lugar a una distribución bastante uniforme en toda la planta del edificio; en cambio, el agua dispersa produce zonas de gran concentración, mientras otras permanecen casi secas.

Estas dos causas de humedad, agua dispersa y freática, pueden hasta cierto punto distinguirse por las manifestaciones exteriores que producen en los muros por encima del nivel del suelo. El examen de tales características es útil, cuando no sea posible efectuar un examen detenido abriendo pozos o zanjas al pie de los muros.

a) caracteres de la humedad debidos al agua dispersa.

1) presenta manifestaciones imponentes, pero muy localizadas, en una sola parte o a un solo lado del edificio;

2) es característica de un solo edificio o un grupo de edificios muy próximos;

3) presenta a menudo oscilaciones anuales en su nivel sobre el suelo; 

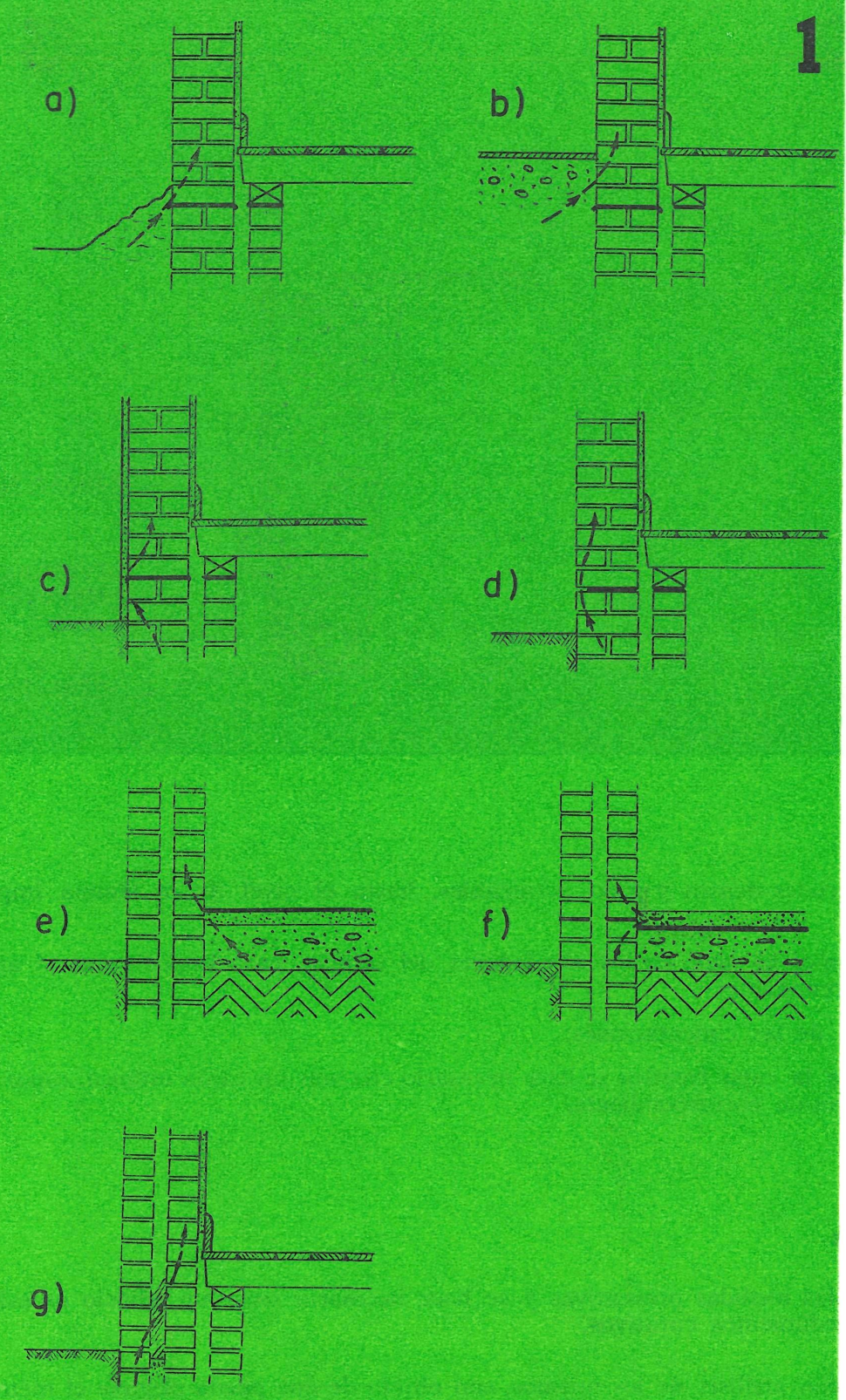

b) caracteres de la humedad debidos al agua freática.

1) ataca al edificio en toda la planta, con perfecta uniformidad, salvo que la estructura esté construida con materiales diferentes;

2) el nivel es máximo para orientaciones expuestas y mínimo para orientaciones muy aisladas;

3) es común a todos los edificios de la misma zona (de aproximadamente igual construcción y antiguiedad);

4) el nivel de la humedad no presenta oscilaciones anuales de importancia.

\section{Soluciones \\ provemtivers}

Es de capital importancia prevenir los desórdenes debidos a la humedad, antes de iniciarse la construcción y efectuar ésta de acuerdo con las medidas proyectadas, pues la reparación posterior encierra a menudo grandes dificultades tanto de orden técnico como económico.

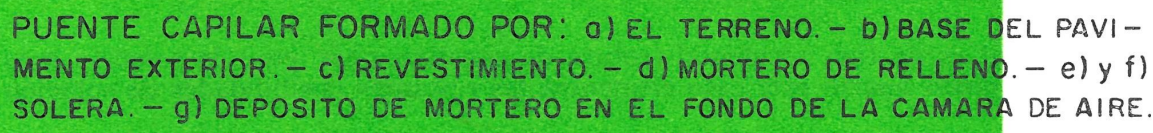

Las disposiciones a adoptar se incluyen normalmente entre las enumeradas a continuación:

- procurar no construir en un terreno anegado de agua;

- drenar las aguas del suelo para alejarlas de las cimentaciones;

- interponer láminas horizontales impermeables en las partes bajas de los muros, a algunos decímetros sobre el nivel del suelo. Es de suma importancia no dejar ningún puente capilar que permita al agua ascender a través del mismo (fig. 1); 


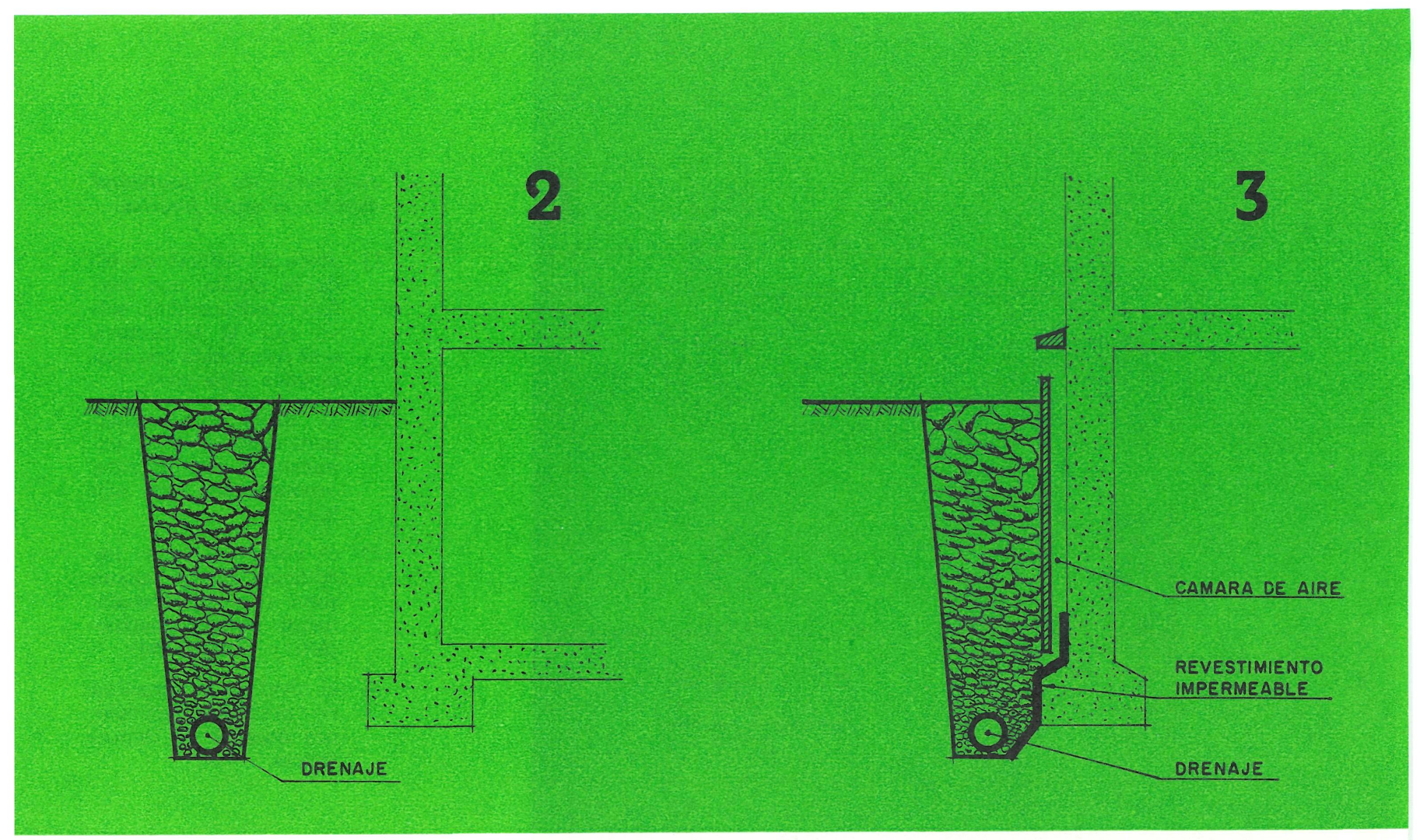

- utilizar en las cimentaciones materiales densos y poco permeables, hasta el nivel de la lámina impermeable;

- recubrir con un revestimiento estanco el suelo en los alrededores del edificio, para impedir que el agua de lluvia penetre en el terreno circundante;

- efectuar un revestimiento estanco de las cimentaciones;

- en caso de sótanos por debajo de la capa freática se hace necesario efectuar un cajón estanco, capaz de evitar la entrada de agua a la presión correspondiente.

\section{Soluciomes cumcitivas}

Cuando por descuido no se han tomado las medidas preventivas y se hace necesario secar un edificio excesivamente húmedo, existe una serie de procedimientos curativos.

Estos se indicarán efectuando un pequeño análisis de los mismos, con objeto de que pueda elegirse el método o combinación de ellos más idónea y de mejor aplicación en cada caso.

Es obvio que si el origen de la humedad es de tipo accidental (rotura de canalizaciones, etc.), la solución más drástica será reparar el desperfecto. Dejando aparte este caso elemental, las restantes posibilidades de solución son las siguientes:

\section{A. Impedir al agua aproximarse a las cimentaciones.}

1. Construyendo una zanja perimetral de desagüe hasta una profundidad mayor que la cimentación, en cuyo fondo descansará un tubo de drenaje. La zanja se rellenará con capas de piedra, cuyos tamaños disminuirán, desde la capa superior a la inferior, de forma que constituyan un filtro sobre el dren (fig. 2).

2. Construyendo una cámara de aire ventilada, entre el terreno y la cimentación, colocando placas especiales prefabricadas de hormigón, cerámica, etc. (fig. 3), o mediante otro procedimiento de ejecución. 
3. Mediante la aplicación de un revestimiento impermeable en la superficie exterior de las cimentaciones (fig. 4). El revestimiento será bituminoso o de mortero impermeabilizado con un hidrófugo de masa

(Si bien estos dos últimos procedimientos son eficaces para impedir la filtración horizontal del agua de lluvia, no parecen del todo recomendables contra la ascensión capilar del agua freática si no van acompañados del drenaje adicional a nivel inferior de las cimentaciones.)

Los drenes serán tubos de arcilla, cemento, etc., con la parte inferior maciza e impermeable y la superior cuajada de pequeños agujeros. Los tubos estarán perfectamente asentados sobre el terreno, que deberá estar al menos nivelado y apisonado o sobre camas o soleras empedradas de fábrica o de hormigón. La pendiente mínima de los drenes será del 1 por $100 \mathrm{y}$ desembocarán en un tubo o colector principal, encargado de conducir las aguas fuera del recinto a desecar, o, en su defecto, a un pozo que se situará en la parte más baja del terreno y se mantendrá a nivel constante mínimo, extrayéndose el exceso de agua por medio de bombas.

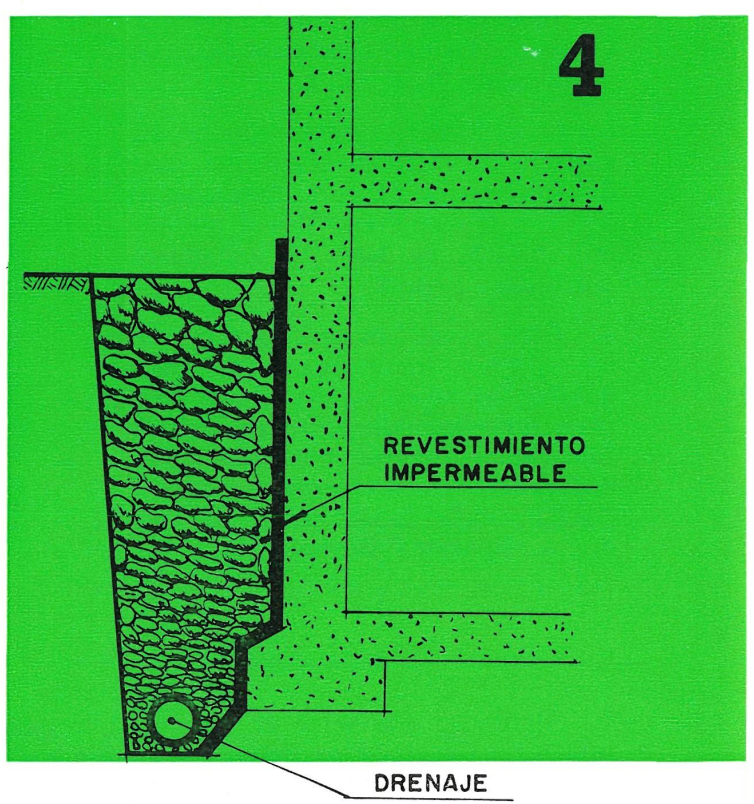

B. Impedir al agua ascender por los muros.

1. Introduciendo láminas impermeables en cortes practicados en los muros por su parte baja (figs. 5 y 6). Las láminas pueden ser: asfálticas, de plomo, cobre, polietileno, cloruro de polivinilo, etc. El vacío existente encima de las placas se rellena con mortero.

Una variante de este sistema, es descalzar el muro a una altura de 30 ó $40 \mathrm{~cm}$ sobre el nivel del suelo y sustituir dos o tres hiladas de ladrillo por otras recibidas con mortero hidrofugado hasta conseguir una barrera horizontal impermeable como en el caso anterior (fig. 7). Los cortes se efectuarán en ambos casos por tramos no consecutivos de 50 a $100 \mathrm{~cm}$ y de forma que nunca esté cortado a la vez más de un tercio de la longitud total del muro.

Este procedimiento resulta totalmente eficaz, pero solamente se aplica a muros poco cargados (casas bajas) de mampostería o ladrillo.

5
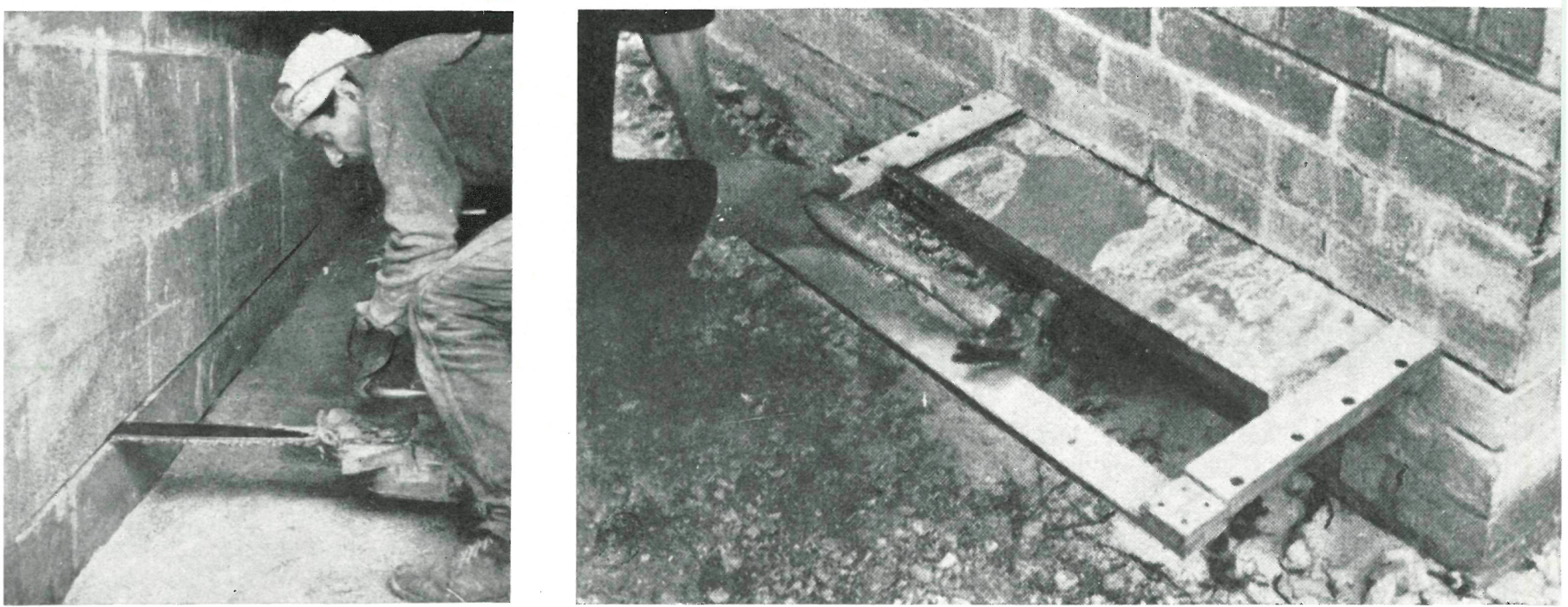
2. Inyectando productos impermeabilizantes en una hilera de orificios practicados en el muro.

Un procedimiento patentado en Inglaterra consiste en abrir una serie de agujeros de $10 \mathrm{~mm}$, distanciados $3 \mathrm{~cm}$, e inyectar en ellos a presión una mezcla de látex de caucho y siliconato de sodio.

3. Otro procedimiento es perforar los agujeros inclinados hacia abajo dentro del muro y verter una solución impermeabilizante que se difunde por gravedad. Este nuevo procedimiento es interesante, mas se desconoce la composición del producto impermeabilizante.

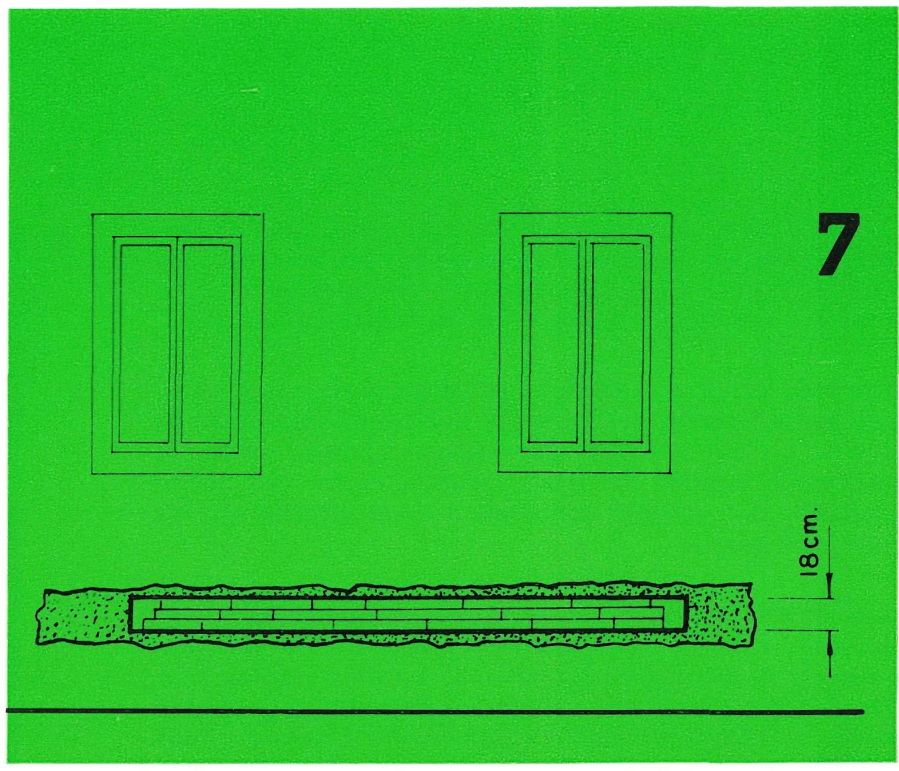

\section{Evaporar el agua absorbida.}

1. Mediante una serie de orificios practicados en el muro inclinados unos $20^{\circ}$ ó $30^{\circ}$ hacia arriba. Existe una serie de artificios patentados para insertar en los orificios y mejorar su rendimiento (tubos de bizcocho poroso, metal, plástico con y sin rejillas interiores, etc.) $(*)$.

Estos procedimientos, aunque aumentan en cierto modo la superficie de evaporación, pueden en algunos casos debilitar la obra en que están aplicados.

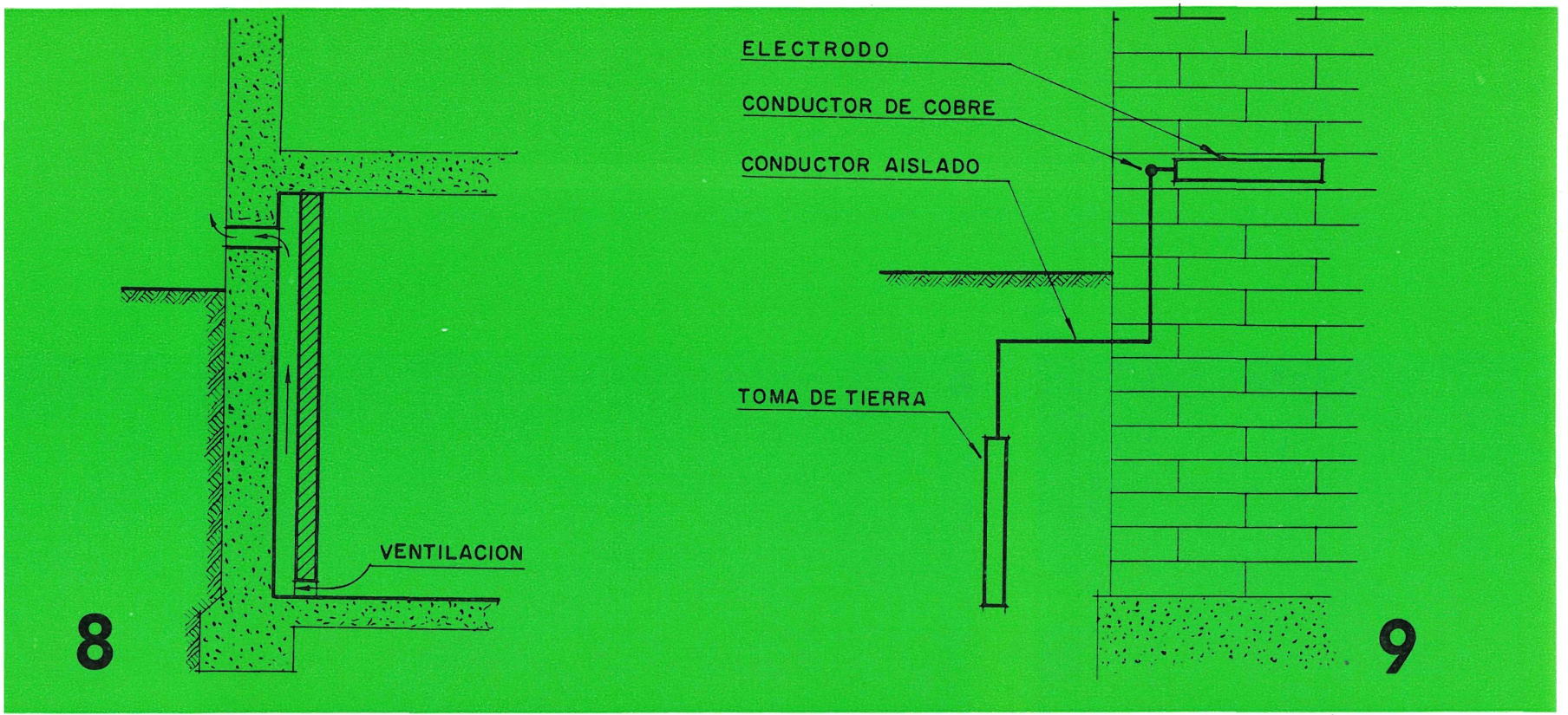

2. Construyendo una cámara de aire ventilada entre el muro húmedo y el revestimiento interior. Este puede ser de placas de amianto-cemento, paneles imputrescibles, etc. La cámara de aire debe tener unos $3 \mathrm{a} 5 \mathrm{~cm}$ de ancho, y las aberturas de ventilación irán en la parte de arriba hacia el exterior y en la de abajo hacia el interior. Las aberturas serán de unos $30 \mathrm{~cm}^{2}$ por metro de revestimiento. Este procedimiento se aplica, sobre todo, a sótanos húmedos (fig. 8).

(*) Ver "Bâtir", núm. 114, julio 1962. 
D. Eliminar el agua por electroósmosis.

Este procedimiento, que es de origen suizo (patente Ernst), se ha modificado posteriormente en otros países como Hungría y Rusia. El fundamento es el siguiente:

Cuando el agua del suelo es absorbida por un muro, aparece una diferencia de potencial entre el suelo (negativo) y el muro (positivo).

Para conseguir la polaridad contraria se establece el siguiente circuito:

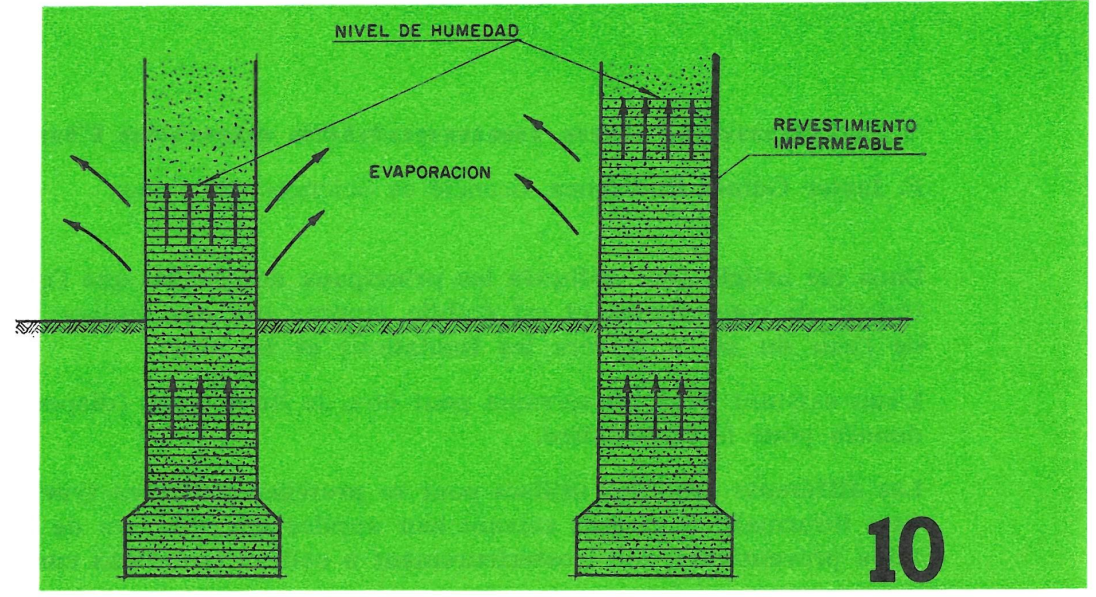

Una serie de electrodos insertados a intervalos regulares en el muro, hasta unos $2 / 3$ de su espesor, se conectan entre sí por medio de una cinta de cobre alojada en una roza horizontal practicada en el muro. La cinta se conecta a su vez, a través de conductores aislados, a varias tomas de tierra constituidas de un metal menos noble (aluminio o cinc) (fig. 9).

El agua, dirigiéndose hacia el polo positivo, ahora situado en el suelo, descenderá desalojando el muro que empezará a secarse.

Este procedimiento parece encontrar buena aceptación en el extranjero, aunque no es todavía muy conocido. Es relativamente fácil de instalar y no requiere gastos de mantenimiento, al mismo tiempo que no altera ni la solidez ni la estética del edificio.

\section{E. Impedir la evaporación interior del agua.}

Por aplicación de un revestimiento estanco, de mortero hidrofugado o pintura impermeable aplicable sobre fondo húmedo.

Este método no es más que un paliativo, pues no elimina el agua absorbida por el muro, sino únicamente la posibilidad de su evaporación interior; con esto el equilibrio (absorción-evaporación) se rompe y el agua se eleva por el muro a un nivel más alto (fig. 10). Es necesario, por tanto, efectuar el revestimiento estanco hasta por encima del nivel máximo a que haya llegado el agua.

No es un método recomendable, pero, algunas veces, es el único de posible aplicación.

\section{Comclusión}

Habida cuenta de las dificultades y coste de las impermeabilizaciones posteriores, la mejor solución es prever éstas en el proyecto y ejecutarlas simultáneamente a la construcción. Cuando se haga necesario adoptar soluciones curativas, es imprescindible determinar con exactitud el origen de la humedad y ejecutar en cada caso el procedimiento o combinación de ellos más conveniente.

\section{Bibliografía}

"L'Assèchement des murs", Centre Scientifique et Technique de la Construction, cahier mensuel num. 9. Bruxelles, 4 sep. 1965.

"Risanamento igienico dei locali umidi" Viovanni Massari. Ed. Ulrico Hopli. Milano, 1952.

"Rising damp in Walls", Building Research Station Digest, n.०27, 2nd series. England, oct. 1962.

"Pliego General de Condiciones varias de la Edificación". Madrid, 1948. 


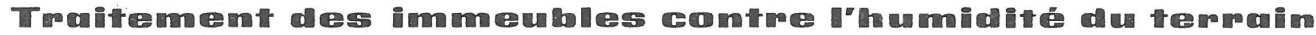

F. Javier Gómez Rodríguez, licencié ès Sciences Physiques

Dans cet article sont indiqués les principaux dommages que l'excès d'humidité peut causer aux matériaux de construction et est établie, comme une des causes fondamentales, l'ascension capillaire de l'eau du sol à travers les fondations et les murs.

L'eau existant dans les terrains peut être de deux types (dispersée et phréatique). Des critères sont donnés pour la reconnaître.

Les différents procédés utilisés pour se protéger contre ce type d'humidités sont ensuite indiqués à titre informatif et classés suivant leur application, soit lors de la construction de l'immeuble (solutions préventives), soit postérieurement à celle-ci (solutions curatives).

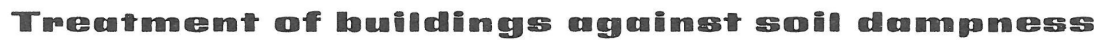

F. Javier Gómez Rodríguez, physics graduate

This paper mentions the main damage that may arise from excessive dampness in building materials. One of the main sources of this humidity is the rising of water through capillarity from the ground, and across foundations and walls.

Soil water may be of two kinds (disperse and freatic), and criteria are given for recognising each type.

As a matter of information, the paper finally mentions the methods that are utilised to counteract the above type of humidity. These methods are termed preventive, when they are applied during the construction of the building; and curative, if undertaken after the building has been completed.

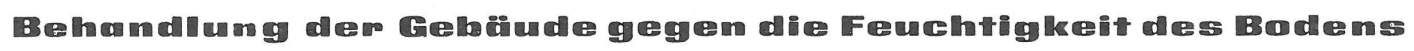

F. Javier Gómez Rodríguez, Dipl. Physiker

Im vorliegenden Artikel sind die wichtigsten Schäden, die wegen der grossen Feuchtigkeit in den Baumaterialien auftreten werden können. Ein der wesentlichen Gründe ist den Kapillaranstieg des Bodenswassers durch die Grundmauern und die Wände.

Das liegende Wasser im Boden kann von zwei Typen (zerstreuetes und phreatic) sein und es bestehen Normen um es zu untersuchen.

In der Folge zeigt man an, die verschiedenen Methoden, die man anwendet, um vor diesem Typ von Näse zu beschüitzen. Sie werden wegen ihrer Anwendung entweder während des Gebäudesbaues (vorsichtige Lösungen) oder nach dem Bauen (heilige Lösungen) eingeteilt. 